\title{
Crossing the Communication Chasm: Challenges and Opportunities in Transitions of Care From the Hospital to the Primary Care Clinic
}

\author{
Nicholas A. Rattray, PhD; Jason J. Sico, MD; LeeAnn M. Cox, MD; Alissa L. Russ, PhD; Marianne S. Matthias, PhD;
} Richard M. Frankel, PhD

Background: Transitions of care from specialty and acute settings to primary care abound. Compared to the continuity in end-of-shift handoffs, care transitions involve provider communication between practices and facilities with their own cultures and bureaucracies. Using the transition from acute care to outpatient primary care for stroke/transient ischemic attack (TIA) patients as a case study, this qualitative research explored communication practices and institutional arrangements among clinical providers responsible for longitudinal management of hypertension.

Objectives: Researchers investigated the barriers and facilitators of effective communication between acute stroke/TIA inpatient and primary care providers at a Veterans Affairs Medical Center.

Methods: A multidisciplinary team conducted consensus-based coding and thematic analysis of semistructured interviews with 21 clinical providers ( 9 with primary responsibilities for inpatient care and 12 with primary responsibilities in outpatient, primary care).

Results: Thematic analysis of responses identified three factors that influenced communication between clinical providers: (1) consistent, concise but complete medication and treatment plans; (2) reliable, standardized discharge documentation; (3) use of multiple modes of communication. Participants identified cultural barriers, including challenges with rotating providers at a teaching hospital and local discharge practices.

Conclusion: Ambiguity about who is being handed off to and time pressures in the acute setting may lead inpatient providers to give lower priority to discharge communication, leaving outpatient providers with low-quality information. While electronic templates have standardized key components of discharge documentation, improvement opportunities remain. Increased awareness of the challenges and opportunities on each side of the care transfer could foster communication practices that systematically account for the information needs of inpatient and outpatient providers.

$S^{i}$ ince The Joint Commission specified a standardized approach for handoffs in its 2006 National Patient Safety Goals, an extensive literature has emerged investigating transitions across the continuum of care and responsibility for patient discharge. ${ }^{1-4}$ While progress has been made in assessing within-unit transitions and end-of-shift handoffs, recent studies have revealed the inherent complexity involved in transitions of care, such as those between inpatient and outpatient care providers. ${ }^{5}$ Recent assessments proposing interventions for improving transitions of care have suggested that novel conceptual frameworks are necessary to move beyond the mechanics of information processing toward underlying motivations, social dynamics, and contextual factors that shape how communication occurs between different types of providers. ${ }^{6}$ Kripalani and colleagues in a systematic review emphasized how incomplete discharge

1553-7250/\$-see front matter

Published by Elsevier Inc. on behalf of The Joint Commission.

http://dx.doi.org/10.1016/j.jcjq.2016.11.007 information, such as conflicting diagnoses, delayed test results, and inaccurate medication plans, creates discontinuities between hospital-based and primary care physicians. ${ }^{4}$ As compared to end-of-shift handoffs where terminology, physical context, and other structural factors are relatively stable, transitions of care typically involve facilities with their own culture and bureaucracy. ${ }^{7}$ Variability in organizational structures, the flow of work, and patient care goals have been identified as potential challenges in transitions of care that cross unit- or facility-based boundaries. ${ }^{8}$ Discontinuities between the inpatient and outpatient settings under the hospitalist model of care require adaptations in discharge communication. ${ }^{\text {? }}$ Given that miscommunication between providers during transfers of care can lead to serious preventable adverse events, research that identifies barriers to communication in transitions of care is critical for ensuring patient safety. ${ }^{10}$

The transition of stroke patients from acute inpatient care to treatment in primary care clinics depends on effective communication. Stroke is an important case to examine care transitions in particular, because postdischarge care for patients 
with stroke, such as hypertension management, is critical. Stroke is the second leading cause of death worldwide and fourth in the United States. ${ }^{11}$ About every 40 seconds someone has a stroke, and every 4 minutes a person dies from one. Hypertension, one of the most common conditions managed in outpatient primary care settings, is the single most important modifiable vascular risk factor for patients with acute ischemic stroke or transient ischemic attack (TIA). ${ }^{12}$ Surprisingly few studies have investigated the dynamics of communication between inpatient and outpatient providers who care for stroke/TIA patients. Evidence suggests that there are persistent deficiencies in the quality and delivery of secondary prevention strategies. ${ }^{13-16}$ Potential barriers to patient care following stroke/TIA include the following: suboptimal follow-up of stroke survivors; inadequate initiation and intensification of antihypertensive medications during hospitalization and outpatient followup; differences in expectations regarding hypertension management in the period immediately after discharge; and poor communication between inpatient specialty providers and outpatient primary care providers (PCPs). ${ }^{17-20}$ Ample evidence of a "vulnerable gap" in the transition of poststroke/TIA patients from inpatient to outpatient settings suggests a need to learn more about how these transitions take place and the thought processes that underlie provider behavior.

We aimed to study how various forms of discharge communication (for example, face-to-face, electronic) affect patient discharge using stroke/TIA patients as a case study in a tertiary Veteran Affairs Medical Center (VAMC). As the largest integrated system in the United States, the Department of Veterans Affairs (VA) has used an electronic health record (EHR) system since 1999. ${ }^{21}$ The VA's adoption of the EHR has transformed clinical communication, leading to increased efficiency but also creating new sources of error $^{22}$ and reduced face-to-face and telephonic communication, which in other industries has been shown to provide the highestquality transfers of information. ${ }^{23}$ We designed a qualitative study focusing on the transition in management of hypertensions for patients with stroke/TIA who were discharged from the hospital to primary care. Thematic analysis of interviews conducted with clinical providers identified facilitators and barriers of effective transitions between care settings, the specific reasons they exist, and strategies that might address communication problems.

\section{METHODS}

\section{Setting}

The study, which received ethics approval from the Indiana University Institutional Review Board, was conducted at the Richard L. Roudebush VAMC, an affiliate of the Indiana University School of Medicine. The medical center annually treats more than 200 stroke/TIA patients. Stroke/TIA patient care was selected as a case study because safe and effective management of hypertension depends on coordinated, cross-service communication.

\section{Provider Sample}

In a traditional academic model, Neurology admits and discharges most stroke/TIA patients in addition to serving in a consulting role to the medicine hospitalist unit. Approximately $75 \%$ of the patients with strokes/TIAs also have a history of hypertension, necessitating medication adjustments at discharge. Blood pressure is managed by teams comprised of an attending physician, residents/interns, medical students, a clinical pharmacist, and a nurse care manager. Although discharge documents are structured templates intended to ensure inclusion of key components (for example, diagnoses, tests, hospital course), they vary considerably and typically lack specific recommendations for ongoing blood pressure management in the transitional period before outpatient care.

Because physicians, nurses, and pharmacists play key roles in patient discharge, we recruited a purposive sample of 21 providers, with representatives from each group. We selected respondents based on their clinical involvement in discharge processes. Participants included 8 physicians (neurologists, residents, internists, and outpatient providers), 8 nurses, and 5 clinical pharmacists. Of the 21 providers, 9 had primary responsibilities in inpatient care, and 12 primarily worked in primary care/outpatient clinics. Participants included 12 women and 9 men (average age: 40) with a median VA experience of 10 years (range: 2-35). A project coordinator experienced in qualitative interviewing conducted a semistructured one-on-one interview lasting 1530 minutes. Key topics included perceptions of hypertension management, communication issues, education/training, and components of optimal discharge summaries. The interview guide was piloted with test participants and refined accordingly (see Sidebar 1 and Sidebar 2). ${ }^{24,25}$ Although patients were recruited through physician referral during their inpatient stay, insufficient sample size and patients' limited cognitive ability to respond led us to focus our analysis on clinical providers. Ethics approval was obtained from the university Institutional Review Board and VAMC Research Committee prior to recruitment.

\section{Data Collection}

Data collection took place between June 2013 and July 2014. Interviews were audio-recorded, professionally transcribed verbatim, checked for accuracy, and then imported into Atlas-ti, a qualitative analysis software tool. ${ }^{26} \mathrm{~A}$ multidisciplinary team analyzed data during a six-month period using immersion/crystallization, an inductive method for identifying themes. ${ }^{27}$ The team consisted of an internist (LC), a neurologist (JS), a qualitative health researcher (RF), and an anthropologist (NR). In the first phase, three team members used a "horizontal pass" ${ }^{27}$ technique to independently identify meaningful segments by reading each transcript in its 


\section{Sidebar 1. Inpatient Clinician Interview Guide}

Section 1: Previous Experience

1. What type of training, if any, have you had in communicating with patients and colleagues about hospital discharge?

2. Please describe your approach to talking with post-stroke/TIA patients and family members about the importance of medication adherence with their hypertension medications.

Section 2: Stroke Specific Communication

3. What are some of the challenges/barriers you experience in communicating with post-stroke/TIA patients who have hypertension at discharge?

4. What are the greatest satisfactions you experience in communicating with and about these patients?

5. What are the challenges you experience in communicating with other inpatient team members (physicians, nurses, PT/OT etc.) about post stroke/TIA patients with ongoing hypertension?

6. What are some of the challenges you experience in communicating with primary care clinicians about post stroke/TIA patients with hypertension?

7. Please describe your role in managing hypertension in post stroke/TIA patients?

—During the acute hospitalization?

-During discharge?

8. If there are specific hypertension management guidelines or recommendations you follow/adhere to for post stroke/TIA patients would you please describe them?

9. In your opinion, what information should the ideal discharge summary contain?

10. In your opinion, what could be improved about the discharge process for post stroke/TIA patients?

Section 3: Teams and Teamwork

11. In what ways has the PACT model helped or hindered care for post-stroke/TIA patients?

12. If you could make one change that would help improve communication with patients and between inpatient and primary care clinicians what would it be?

13. Anything you'd like to add?

entirety. Consensus on patterned themes observed in the data emerged from iterative discussion about variation across interview participants and deviant cases. In the second phase, the anthropologist coded each transcript to identify quotations about barriers and facilitators to communication. Finally, matrices depicting recurring barriers and facilitators among different sets of providers were discussed among the multidisciplinary team. ${ }^{28}$

\section{Sidebar 2. Outpatient Primary Care Clinician Interview Guide}

Section 1: Previous Experience

1. What type of training, if any, have you had in communicating with patients and colleagues about hospital discharge?

2. Please describe your approach to talking with post-stroke/TIA patients and family members about the importance of medication adherence with their hypertension medications.

Section 2: Stroke Specific Communication

3. What are some of the challenges/barriers you experience in communicating with post-stroke/TIA patients who have hypertension and are following up in primary care?

4. What are the greatest satisfactions you experience in communicating with and about these patients?

5. What are the challenges you experience in communicating with other outpatient team members (physicians, nurses, PT/OT, etc.) about post stroke/TIA patients with hypertension?

6. What are some of the challenges you experience in communicating with primary care clinicians about post stroke/TIA patients with hypertension?

7. Please describe your role in managing hypertension in post stroke/TIA patients?

-During the acute hospitalization?

-During discharge?

8. If there are specific hypertension management guidelines you follow/adhere to within one month for post stroke/TIA patients would you please describe them?

9. In your opinion, what information should the ideal discharge summary contain?

10. In your opinion, what could be improved about the discharge process for post-stroke/TIA patients

Section 3: Teams and Teamwork

11. In what ways has the PACT model helped or hindered care for post-stroke/TIA patients?

12. If you could make one change that would help improve communication with patients and between primary care clinicians and inpatient clinicians or team members what would it be? 


\section{RESULTS}

We report on factors that shape variation in communication during transitions of care in two areas: providerprovider interactions and cultural issues. Although interview questions primarily focused on stroke/TIA care and hypertension management, participants consistently reported on facilitators and barriers related not just to stroke/TIA but also to care transitions more generally. We have interpreted examples and representative quotations, chosen by consensus, to classify these themes into barriers (Table 1) and facilitators (Table 2) to effective transitions of care. Edited excerpts of some of these quotations are provided throughout the Results section.

\section{Factors Influencing Provider-Provider Communication}

Analysis of responses identified three factors that influenced communication between clinical providers: (1) consistent, concise but complete medication and treatment plans; (2) reliable, standardized discharge documentation; and (3) use of multiple modes of communication. There was agreement among respondents that when these criteria were met communication was facilitated. By contrast when they were absent it led to poor quality and clarity of discharge information. Each factor is discussed in detail below.

1. Consistent, Concise but Complete Medication and Treatment Plans. PCPs described the challenges of lack of reliability in the discharge information they received, coping with asynchronous anonymous communication, and lack of rationale about discharge planning, treatment, and medication changes. Although discharge summaries utilized evidenced-based templates to ensure inclusion of all necessary components, respondents reported that there is little attention to ensuring their quality, resulting in a great deal of variability and lack of trustworthiness. Providers primarily attributed the wide variation in quality to the "copy and paste" technique of documentation, which was seen as increasing the volume of information but degrading its reliability, and thus usefulness. ${ }^{29}$ Two attending physicians who had each worked for more than a decade in VA outpatient care expressed ideas about areas to improve discharge processes:

What's happening now is that you usually don't get that clear of an assessment. Usually it'll be something like they'll cut and paste from the admission note, so it'll say, like, presumed, stroke or maybe they'll say stroke but they won't say, "I think that this stroke is because this patient had cocaine intoxication." (PCP, P6)

Discharge planning needs to happen early in the hospitalization-follow-up providers need to be contacted so that they understand the plan and can discuss it with the hospital providers along with any needed arrangements for the patient. Some of the arrangements are medical arrangements like appointments and tests, and other arrangements are like home accommodations, like does the patient need a home nurse and when should the patient come back for a medical evaluation and which specialists need to see that patient again, what medications should be taken for how long. (PCP, P9)

These providers suggested that the ideal discharge summary would include a clear assessment of symptoms, including stroke etiology, impact or extent, and a follow-up plan.

2. Reliable, Standardized Discharge Documentation. Inpatient and outpatient providers described the format and locations of various provider records as barriers. Providers expressed frustration with variations in discharge documents:

Fragmentation and a lack of consistency between these documents about what really happened or what's needed or what's going to happen next [because] so many documents have to be filled out by different people, between nurses, doctors, and pharmacists, that it leads to potential risks for patients. (PCP, P11) There's always a discharge summary but there isn't any particular quality control. The medication list is an area of particular difficulty because frequently there's a discrepancy between the discharge medications and the discharge instructions about the medications. We need a way to decrease this sort of error. (PCP, P15)

Thus, excessive information may be misleading, such as when medications taken prior to hospitalization and medication prescribed at discharge are not clearly or consistently differentiated. Participants reported that information necessary for assessment and treatment was often lost amid a string of differential diagnoses, normal test results, or prewritten "coding" phrases that were time consuming to review. Participants perceived increased reporting and compliance requirements as adding unnecessary complexity and redundancy to documentation.

3. Provider Use of Multiple Modes of Communication. A key facilitator of effective transitions of care is having both the time and willingness to communicate outside the EHR. An inpatient physician (P18) suggested that a personal phone call or in-person meeting with an outpatient provider could be beneficial because solely relying on discharge summaries leads to unwanted interpretation. Yet inpatient clinicians also struggled to readily identify a patient's appropriate provider and a direct contact number. Time constraints discourage interpersonal communication. One attending physician pointed out:

There isn't time for [a phone call] since it's not planned into my schedule. If I do call a doctor, I'm interrupting that person's work-If he or she calls me, he's interrupting my day. We need a better way to plan ahead for these conversations to happen. (PCP, P9)

Despite being identified as ideal by inpatient and outpatient physicians, phone, e-mail, and other forms of personal communication are infrequently used. Subsequently, busy outpatient nurses and physicians attempt to use a "best guess" strategy to interpret vague, ambiguous, 
Table 1. Key Barriers to Effective Transitions of Care

Type of Interaction/Issue $\quad$ Barrier $\quad$ Examples $\quad$ Representative Quotations

Inpatient Provider

-Outpatient Provider

(1) Overreliance or

exclusive use of EHR

for provider

communication

(2) Lack of accuracy

and relevance of

information about

medication and

treatment plans

(3) Lack of accuracy, reliability and completeness of discharge

documentation in the EHR

\section{Cultural}

(1) Challenges with rotating providers at a teaching hospital

1a. "Convoluted" system of changeovers inherent in teaching hospitals

3. Discrepancies between discharge medications and discharge instructions;

poor quality of notes

2. Rationale about treatment or medication plan not supplied by inpatient clinician
(2) Cultural models for transitions of care

3) Local practices of discharge 1b. Medical students are not trained in standardized methods of discharge communication

2a. Cultural practices that rely on communication solely through EHR

2b. Transitions of care are conceived as serial exchange rather than as a shared responsibility

3a. Primary care provider unaware of patient hospitalization 3b. Time constraints limit quality (limited comprehension assessment; ex: teach backs) of discharge note
"We can pull up who is on the call list [in the EHR], and I'd say that $50 \%$ of the time that's just completely wrong. So you'll be paging a pager and they'll page with 'What the heck,' and they will say 'No, I'm not on call tonight.'" (Neurology RN, P4)

"As a primary care physician, I feel sometimes the specialist may sort of drop the ball and default to primary care ... there probably needs to be good communication and coordination of follow-up between primary care and neurology." (PCP, P7)

"The ideal discharge summary would contain an assessment of the etiology of the stroke and a specific plan for follow-up in the outpatient setting, including a plan for when the patient needs to be seen in the clinic. What's happening now is you usually don't get that clear of an assessment. Usually it'll be something like they'll cut and paste from the admission note, so it'll say, like, presumed, stroke or maybe they'll say stroke but they won't say, 'I think that this stroke is because this patient had cocaine intoxication, right?' Or, you know, whatever the problem is." (PCP, P6)

"There's always a discharge summary, but there isn't any particular quality control. So the information that's in them and the quality of the information that's in them varies widely. The medication list is an area of particular difficulty because frequently there's a difference or discrepancy between the discharge medications and the discharge instructions about the medications ... we need a way to decrease this sort of error margin." (PCP, P15)

"It's a patient by patient thing. It depends on how well the notes are written, you know. Some of them, they have great notes in there and I can get all the information I need out of the note. That's inpatient doctors. Some of my doctors don't write great notes, you know, they don't. When you see a note that wasn't real detailed or there is something missing, I wish they would have written a better note, because then I end up having to bother the doctor." (RN, P10)

"I think our system is kind of convoluted and we have a lot of changeovers that makes it difficult to pinpoint who is actually attending —Now I am being very general on that, not specific to the stroke-sometimes you think you are talking to the right physician, they will say 'oh, no that's, I am not responsible for that, you need to go to another service for that particular question.' I think it just the uniqueness of us being a teaching facility and that, I think that makes even more challenging." (Inpatient RN, P12)

"You learn by seeing and doing." (Resident, P25)

"Can't remember any specific training; I've had on-the-job training, I think, and that has been years but nothing specific, no workshops or seminars or anything like that that I can recall." (PCP, P15)

"Having to rely on [the EHR], things may not always be put in in a timely manner... The providers don't always have their day's note in yet." (Inpatient Pharmacist, P2)

"We talk a lot about handoff, and there is no hand off ... the current handoff is more like a punt,, , I am going to kick it to you and ... it's your ball ... it is going to require a cultural change where, as part of the discharge process, there is some sort of handoff and it is some sort of communication." (PCP, P15)

"My role in [hypertension management] is zero because in our facility's cultural scheme the hospital team does not call me if my patient is admitted to the hospital. And they also don't call me when the patient is discharged and so therefore I follow up with the patient following discharge. It doesn't have to be that way. I think you know certainly the hospital team has the primary care in the inpatient setting and they have the responsibility. One could ask whether there's some benefit to coordinating with the outpatient doctors. I think there probably is some benefit that could be gained. It almost never happens. I can't even think of the last time anyone's called me about one of my patients who was admitted." (PCP, P9)

"There isn't time for [a phone call] during the day, it's not planned into my schedule and if I do call a doctor ... I'm going to be interrupting that person's work and that person's day... If he or she calls me he's interrupting my day. ... what we need is a better way to plan ahead for these conversations to happen." (PCP, P9) 
Table 2. Key Facilitators of Effective Transitions of Care

\begin{tabular}{|lll}
\hline Type of Interaction Issue & \multicolumn{1}{c}{ Facilitator } & \multicolumn{1}{c}{ Examples } \\
\hline Inpatient Provider & (1) Utilization of multiple & 1. Face-to-face, phone or \\
-Outpatient Provider & modes of & e-mail communication \\
& communication in & between inpatient \\
& addition to EHR & providers and PCPs \\
& & within one week of \\
& & discharge
\end{tabular}

(2) Agreement between inpatient and outpatient providers on

hypertension guidelines and medication plan

(3) Complete, concise, and accurate discharge summaries and clinical notes in the EHR

(1) Standardized approach to teaching nurses, pharmacists, and residents about patient discharge

(2) Shifts in attitudes, values, and behaviors about the nature of the transition process (3) Improved use of multiple communication channels

\section{2a. Standardized} communication between Neurology and outpatient PCPs

$2 b$. Outpatient providers adhere to blood pressure recommendations from Neurology

3. Specific training for residents that reframes discharge documentation as bridge between inpatient and outpatient care

1. Providers educated on discharge

communication

\section{Shared responsibility} for bidirectional communication

\section{Functioning} "teamlets" in Patient Aligned Care Team (PACT) (patient-centered medical home model)
Representative Quotations

"It is like a godsend . . . when I am attending on the inpatient service, I try to encourage my medical students and residents to communicate with the PCP just to try and improve that communication because it is a huge gap; it is assumed the PCP is going to read the Discharge Summary and the PCP will understand what happened and all the changes and additions and, to some extent, that is true that the electronic medical record facilitates some sort of transition; but by itself, it is inadequate and it could be really improved with a phone call or e-mail or some sort of outreach." (PCP, P15)

"I am not aware of a standard protocol. I don't think we have, like for our congestive heart failure patients, when they're discharged we have a special button that we push on in the postdischarge follow-up phone call template. I don't think we have that for stroke patients, so that would be something that would be really helpful." (Outpatient RN, P17)

"During the acute hospitalization we adhere strictly to the guidance provided by the neurology consultant- depending on their recommendations and the context of the specific patient case-we adhere to the recommendations offered by that team, in the sense of managing with the blood pressure to a degree that they wish and that includes heart rate management, that includes medication management in coordination with their recommendations whether it's blood pressure, anticoagulants, or whatever they deem necessary that's important to the management of the TIA patient." (PCP, P11)

"We need to stop going through residents when acute issues are occurring ... they need to learn, but they also need not to be barriers to direct communication between attending [physicians] ... rather than having a process that involves communication with intermediaries, it should be a direct warm handoff and communications between experienced knowledgeable providers who are ultimate[ly] responsible for those veterans rather than those who have no ownership or longitudinal memory." (PCP, P11)

"A lot of the staff nurses have very little knowledge and/or training about strokes so somehow we became the designated stroke floor, but with no extra education... Training needs to be ongoing, like there needs to be extra in-services, if we are going to be dedicated stroke floor." (Inpatient RN, P19)

"In the transition of care ... just coordinating a good comprehensive EMR documentation that allows the next provider to follow through with the acute changes that have occurred . . . is the best we can do." (PCP, P11)

"The PACT model has helped. I know my patients that have had TIAs or strokes, I know who they are, my doc knows who they are, we're able to communicate, the two of us, along with the patient. We're just familiar with their history, familiar with their concerns, so I think it just improves continuity of care for the patient." (Outpatient RN, P17)

"Philosophically, I believe Team-based care is better than physician-driven solo care. So you need for optimal stroke care, stroke/TIA/hypertension management, you need multidisciplinary; you need more eyes; the more eyes the better, a team that improves continuity, that improves access to care, which are important concepts that the PACT model espouses." (PCP, P15) 
or missing discharge information provided by their inpatient colleagues.

\section{Cultural and Systems Barriers to Managing Transitions of Care}

Three systems-level or cultural factors to managing care transitions were identified, (1) challenges with rotating providers at a teaching hospital, (2) cultural models for transitions of care, and (3) local discharge practices.

1. Challenges with Transient Providers at a Teaching Hospital. Participants discussed the challenges inherent in teaching hospitals where medical interns and residents play a pivotal but transient role. An inpatient nurse (P12) explained how a "convoluted system of changeovers" at the end of shifts can make it difficult to identify the appropriate attending physicians and residents. Multiple residents share responsibility for a single patient: One resident may admit the patient, a second resident may care for him or her during the hospitalization, and a third resident may complete the discharge documentation in the EHR. A majority of inpatient and outpatient providers confirmed that training in effective discharge procedures is lacking, leading to uncertainty about the components of a highquality discharge summary. Although some respondents received limited training in discharge communication in medical school, most learned by "seeing and doing." Some providers suggested that greater involvement in discharge documentation by attending physicians and nurse practitioners might be helpful. An attending physician discussed how discontinuities in resident rotations can hinder continuity in patient care:

We need to stop going through residents when acute issues are occurring. They need to learn, but they also need not to be barriers to direct communication between attending [physicians] ... rather than having a process that involves communication with intermediaries, it should be a direct warm handoff between experienced knowledgeable providers who are ultimate[ly] responsible for those veterans rather than those who have no ownership or longitudinal memory. Nurse practitioners are pulled into those discussions because they do provide continuity in longitudinal care, whereas residents tend not to. (PCP, P11)

\section{Models for Transitions of Care and Local Culture.}

Two PCPs discussed how improving transitions of care would involve shifts in the medical center culture. One attending physician reflected on alternative ways of thinking about transfers of care by applying metaphors from American football:

We talk a lot about handoff, and there is no handoff ... the current handoff is more like a punt.... I am going to kick it to you and ... it's your ball ... it is going to require a cultural change where, as part of the discharge process, there is some sort of handoff and it is some sort of communication. I think that the best communication is face-to-face. That is often not feasible, but a phone call would be second best, I would say, and, then, third would be some sort of electronic communication like an e-mail, a text message. (PCP, P15)

The sports analogy suggests that rather than two players on the same team passing the ball, the current practice is to "punt" responsibility from the inpatient team to another team of PCPs. Two PCPs suggested that while the facility's model of care does not require inpatient providers to advise PCPs when their patients are hospitalized, inpatient providers should play more of a role in coordinating between neurology and outpatient clinics:

My own role in managing hypertension is zero because in our facility's cultural scheme the hospital team does not call me if my patient is admitted to the hospital or discharged. Therefore I follow up with the patient following discharge-It doesn't have to be that way. Certainly the hospital team has the primary care in the inpatient setting. One could ask whether there's some benefit to coordinating with the outpatient doctors- there probably is some benefit. I can't even think of the last time anyone's called me about one of my patients who was admitted. That's different in other hospitals, so that's why I said cultural scheme. (PCP, P9).

As a primary care physician, I feel sometimes the specialist may sort of drop the ball and default to primary care. There probably needs to be good communication and coordination of follow-up between primary care and neurology. (PCP, P7)

These PCPs call attention to areas of improvement that require broader changes between units rather than individual shifts in behavior. Other PCPs attributed communication barriers to wide variations in familiarity with patients. Compared to inpatient providers who interact with a patient for 24-48 hours, outpatient providers had long-standing relationships with patients, enabling them to tailor treatment plans based on the patient's goals rather than reliance on providers' judgment or adherence to clinical guidelines.

3. Local Discharge Practices. Participants made clear that communication through the EHR is necessary, but by itself may be ineffective for all transitions between inpatient and outpatient care. Inpatient and outpatient providers acknowledged that PCPs should be made aware that their patient was hospitalized. Participants suggested reorganizing EHR notes by clinical service rather than chronologically for ease of use and to highlight medication changes. A pharmacist suggested adding PCPs as cosigners on discharge documents. One outpatient nurse with 20 years of experience (P13) suggested that the most important change would be that each medical specialty had one "live person" to contact during business hours. Outpatient providers also suggested that the communication was greater among "teamlets" in the patient-centered home model (teamlets include four members: PCP, registered nurse care manager, licensed practical nurse, medical support assistant). ${ }^{30,31}$ Outpatient providers suggested that in-person, phone, or e-mail communication is "highly 
appreciated." Because communication beyond the EHR is rare, when it does occur.

It is like a godsend ... when I am attending on the inpatient service, I try to encourage my medical students and residents to communicate with the PCP just to try and improve that communication because it is a huge gap. It is assumed the PCP is going to read the Discharge Summary, and the PCP will understand what happened and all the changes and additions and, to some extent, it's true that the electronic medical record facilitates some sort of transition. But by itself, it is inadequate and could be really improved with a phone call or e-mail or some sort of outreach. (PCP, P15)

\section{DISCUSSION}

We conducted a qualitative pilot study to better understand the communication challenges faced by providers on both sides of the transfer of care for patients with stroke/ TIA from inpatient to outpatient settings. As a qualitative study it was not designed to draw generalized conclusions. Research utilizing larger qualitative and/or quantitative samples from a larger number of institutions will be necessary to confirm or reject our findings. Examining the perspectives of medical providers responsible for managing hypertension for stroke/TIA patients offers a window into the complex interpersonal interactions involved in delivering integrated care across time and space. Despite our focus on stroke/ TIA patients, local cultural and contextual barriers and challenges to the discharge process outweighed specific concerns about transitions of care for patients with cerebrovascular disease. As one of the few qualitative studies that examine multidisciplinary provider perspectives across the care continuum, ${ }^{32}$ this study substantiates prior research that demonstrates how ambiguities and inconsistences in communication from inpatient providers created uncertainty and frustration for PCPs. ${ }^{4,93}$ This was true despite the fact that residents rotated on inpatient and outpatient services and have experienced both roles. While we recognize that it may be unrealistic to expect busy providers to engage in all of the communication processes that they described in interviews as optimal, our analysis suggests several potential improvement opportunities for discharging post-stroke/ TIA patients and discharge communication in general: (1) standardized, mutually understood guidelines for communication and hypertension management between neurology and primary care; (2) modifications to discharge summary templates that require specific details about changes to medication regimens during hospitalization, rationale for treatment decisions, and proposed treatments; and (3) standardized mechanisms to ensure that PCPs are informed about inpatient hospitalizations for their patients. Table 3 (page) outlines current approaches and relevant interventions that address the three major challenges we identify.

Despite the fact that studies have shown that hospital and PCPs generally share consensus on what constitutes a high-quality discharge summary, ${ }^{34}$ communication of discharge information remains a source of discontinuity between the acute and chronic phases of care, leading to lower quality and adverse clinical outcomes. ${ }^{4}$ In the area of stroke, more effective discharge communication could lead to significant reductions in hospital readmission. ${ }^{35}$ Johnson et al. suggest that inpatient providers typically adopt a "hospital centric" view with an overreliance on the patient as "information courier." ${ }^{36}$ Inpatient and outpatient providers appear to lack a sense of "shared common ground" 37 that would facilitate collaboration about transitions of care. This may stem from disjointed work flows and a lack of cognitive connection that trainees make between their inpatient and outpatient roles. ${ }^{38}$

Our findings also align with research on the unintended consequences of the EHR. ${ }^{39}$ One cost of EHR communication is the "checkbox mentality" of templates that may discourage providers from developing a clear picture or narrative about individual patients. ${ }^{40,41}$ This may in part be driven by EHR templates designed for easy data extraction for billing and coding, but not for inclusion of a diagnostic or therapeutic rationale connecting the discharge information to the outpatient provider's task. Studies of the VA's EHR have suggested that the practice of copying and pasting notes also leads to both "benign" and "misleading" errors, as well as to a lack of updates regarding patient conditions, and to redundant information, ${ }^{42}$ which may point to inherent limits of EHRs compared to paper records. ${ }^{22}$

Awareness of the significance of discontinuities in care has been heightened with the recognition that medical error is the third leading cause of death in the United States. ${ }^{43} \mathrm{~A}$ recent systematic review found that although organizational culture and standardized measures of continuity of care are key factors in the quality and safety of care transitions, few interventions focus on changing the attitudes of care providers or offering specific guidance on producing relevant, timely discharge information. ${ }^{5}$ In a study of first-year internal medical residents, approximately one in three discharge summaries were inaccurate in the areas of medication lists, medication changes, and follow-up plans. ${ }^{44}$ Interventions aimed at improving communication may cultivate a "pay-it-forward" or anticipatory management sensibility, ${ }^{45}$ whereby inpatient providers conceptualize their relationship with PCPs as a form of indirect reciprocity. ${ }^{46}$ Rather than viewing the transition of care as a mechanical process, in this model the inpatient provider should ask, "What would I want and need as the primary outpatient provider who will be providing the next steps in care for this patient?" Establishing two-way communication would likely generate more interest in altruistic acts that can foster cooperation across clinical domains. ${ }^{47}$ Although medical residents learn how to practice in both inpatient and outpatient settings, they may not always appreciate the fluidity of their different roles. Explicitly connecting the two may improve the quality of information contained in the discharge summary 
Table 3. Implications for Hospitals and Primary Care Providers (PCPs) Regarding Communication Across Transitions of Stroke Care

\begin{tabular}{|c|c|c|}
\hline Key Challenge & Addressing Gaps in Current Approach & $\begin{array}{l}\text { Effective Interventions Reported } \\
\text { in the Literature }\end{array}$ \\
\hline $\begin{array}{l}\text { 1. Consistent, concise but complete } \\
\text { medication and treatment plans }\end{array}$ & $\begin{array}{l}\text { - For stroke patients requiring hypertension } \\
\text { management, hospitalist physicians include } \\
\text { treatment rationale or clarity about } \\
\text { medication } \\
\text { "Two minute" summaries of treatment and } \\
\text { discharge plan }\end{array}$ & $\begin{array}{l}\text { I-PASS }{ }^{1-3} \\
\text { Bundled set of interventions emphasizing } \\
\text { illness severity, patient summary, action items, } \\
\text { situation awareness and contingency plans, } \\
\text { and synthesis by receiver } \\
\text { TRACER }^{4} \\
\text { 2-week resident rotation in which trainees } \\
\text { follow patients into post-acute care, enabling } \\
\text { insights into issue faced by PCPs }\end{array}$ \\
\hline $\begin{array}{l}\text { 2. Reliable, standardized discharge } \\
\text { documentation }\end{array}$ & $\begin{array}{l}\text { - Provide hospitalists access to electronic } \\
\text { health information that PCPs require } \\
\text { - Regular feedback given by PCPs and/or } \\
\text { attending physicians on discharge } \\
\text { communication }\end{array}$ & $\begin{array}{l}\text { Resident-Directed Quality Improvement } \\
\text { Program on Care Transitions } \\
\text { Incentive-based program with education on } \\
\text { care transitions, standardized documentation, } \\
\text { audit and feedback, and rapid data analysis. } \\
\text { Audit and Feedback on Discharge } \\
\text { Summary } \\
\text { Pre-post intervention study with targeted } \\
\text { feedback for fellows resulted in improvement } \\
\text { in discharge documentation }\end{array}$ \\
\hline $\begin{array}{l}\text { 3. Use of multiple modes of } \\
\text { communication }\end{array}$ & $\begin{array}{l}\text { - Include a note and phone number to } \\
\text { person receiving transition of care } \\
\text { - Training that emphasizes how local cultures } \\
\text { and context affect communication regarding } \\
\text { transitions of care }\end{array}$ & $\begin{array}{l}\text { Face-to-Face Is Optimal for Handoffs } \\
\text { Patterson and colleagues }{ }^{7} \text { have reviewed the } \\
\text { literature on handoffs in a number of high } \\
\text { reliability industries and concluded that face- } \\
\text { to-face handoffs have the highest accuracy } \\
\text { and reliability. } \\
\text { TeamSTEPPS } \\
\text { Toolkit aimed at building competency in team } \\
\text { leadership, situation monitoring, mutual } \\
\text { support, and communication }\end{array}$ \\
\hline \multicolumn{3}{|c|}{$\begin{array}{l}\text { References: } \\
\text { 1. Starmer AJ, et al. I-pass, a mnemonic to standardize verbal handoffs. Pediatrics. 2012;129:201-204. } \\
\text { 2. Starmer AJ, Landrigan CP. Changes in medical errors with a handoff program. N Engl J Med. 2015 Jan 29;372:490-491. } \\
\text { 3. Starmer AJ, et al. Development, implementation, and dissemination of the I-PASS handoff curriculum: A multisite educational inter- } \\
\text { vention to improve patient handoffs. Acad Med. 2014;89:876-884. } \\
\text { 4. Meade LB, et al. TRACER: An "eye-opener" to the patient experience across the transition of care in an internal medicine resident } \\
\text { program. J Community Hosp Intern Med Perspect. } 2015 \text { Apr 1;5:26230. } \\
\text { 5. Kalanithi L, et al. The effect of a resident-led quality improvement project on improving communication between hospital-based and } \\
\text { outpatient physicians. Am J Med Qual. 2013;28:472-479. } \\
\text { 6. Dinescu A, et al. Audit and feedback: An intervention to improve discharge summary completion. J Hosp Med. 2011;6:28-32. } \\
\text { 7. Patterson ES. Communication strategies from high-reliability organizations: Translation is hard work. Ann Surg. 2007;245:170-172. } \\
\text { 8. King HB, et al. TeamSTEPPSTM: Team strategies and tools to enhance performance and patient safety. In Henriksen K, et al. editors: } \\
\text { Advances in Patient Safety: New Directions and Alternative Approaches, vol 3: Performance and Tools. Rockville, MD: Agency for Health- } \\
\text { care Research and Quality, 2008. Accessed Nov 22, 2016. http://www.ahrq.gov/downloads/pub/advances2/vol3/advances-king_1.pdf. } \\
\text { 9. Thomas L, Galla C. Building a culture of safety through team training and engagement. BMJ Qual Saf. 2013;22:425-434. }\end{array}$} \\
\hline
\end{tabular}

as well as facilitating interpretation of the information by the PCP.

More research connecting inpatient and outpatient roles in resident training, specific challenges that relate to stroke/ TIA care, and approaches to educating providers constitute next steps in a research agenda. Despite widespread awareness of the negative consequences of poor communication in handoffs and transitions of care, little research has identified best practices. ${ }^{7,48,49}$ Authors such as Hilligoss and
Cohen $^{50}$ and Horowitz and colleagues ${ }^{51}$ suggest that education for effective handoffs incorporate direct observation and feedback or handoff behaviors, use of reflective practice as a way to inculcate vigilance, and attention to differences in handoff types (for example, within-unit handoffs [between trainees who are largely known to one another] and betweenunit transfers of care [between trainees or attending physicians who are strangers to one another]). We are developing a handover tool for residents and attending physicians 
discharging hospitalized patients based on an earlier study of anticipatory management in end-of-shift handoffs. ${ }^{45}$ The tool systematizes the hospital course content into a logical series of steps that encourages perspective taking and holistic, narrative-based modes of communication. ${ }^{52}$ Further evaluation is necessary, but the tool has been tested on one cohort of residents and has been recommended on a trial basis by the graduate medical education division as a model for improving discharge communication across disciplines.

\section{Limitations}

This study offers insights into cultural factors that inpatient and outpatient providers perceive as influencing communication during transitions of care. Findings from the small sample drawn from a single VAMC are not meant to generalize to other medical systems nor to providers caring for all types of cerebrovascular patients. Study participants may have focused on generic issues faced by their teams rather than their individual perspectives on effective communication practices for such patients. Similarly, stroke/TIA patients may represent a small proportion of their patients and thus may be subsumed into a generic set of practices. Finally, because interviews with patients were not included in our analysis, our findings are limited to the provider perspective alone. Future studies may also incorporate direct observation of activities such as EHR data entry or face-toface communication of discharge summaries.

\section{CONCLUSION}

Drawing from interviews with inpatient providers and PCPs, we found that effective transitions of care require clear, unambiguous communication to reduce the risk of misunderstanding that could lead to patient harm. Inpatient providers, constrained by the rapid pace of acute care and duty hour restrictions, may not prioritize writing concise, "high quality" discharge notes and instructions with the end users in mind. On the receiving end of the discharge process, outpatient providers face difficulties sifting through disorganized, redundant information. Increasing trainees' and practicing physicians' awareness of the challenges and opportunities that lie on both sides of transfers of care may be helpful in creating a culture in which the anticipated information and management needs of both inpatient and outpatient providers are systematically taken into account.

Acknowledgments. The authors thank the health care providers who participated in this study. The authors particularly appreciate the contributions of Dr. Alan Zillich, Angela Harris, Dr. Siying Chen, and Amy Strasburger. Funding. This research is based on grants funded by the US Department of Veterans Affairs, Veterans Health Administration, Office of Research and Development, Health Services Research and Development (VA HSR\&D) Quality Enhancement Research Initiative RRP 12-200 and CDA 11-214. The views expressed in this article are those of the authors and do not necessarily represent the views of the Department of Veterans Affairs or the US government.
Nicholas A. Rattray, PhD, is Research Scientist, Center for Health Information and Communication, Richard L. Roudebush Veterans Affairs Medical Center (VA CHIC RVAMC), Indianapolis, and Adjunct Assistant Professor, Department of Anthropology, Indiana University-Purdue University Indianapolis (IUPUI). Jason J. Sico, MD, MHS, is Assistant Professor, Departments of Neurology and Internal Medicine, Yale University School of Medicine, New Haven, Connecticut, and Director of Stroke Care, VA Connecticut Healthcare System, West Haven. LeeAnn M. Cox, MD, is Assistant Professor of Clinical Medicine, Indiana University School of Medicine, Indianapolis, and Hospitalist, RVAMC. Alissa L. Russ, PhD, is Research Scientist, VA CHIC RVAMC, and Assistant Professor, Department of Pharmacy Practice, Purdue University, West Lafayette, Indiana. Marianne S. Matthias, PhD, is Research Scientist, VA CHIC RVAMC, and Associate Professor, Department of Communication Studies, IUPUI. Richard M. Frankel, PhD, is Research Scientist, VA CHIC RVAMC, and Professor of Medicine and Geriatrics, Indiana University School of Medicine. Please address correspondence to Nicholas A. Rattray, nrattrray@iupui.edu.

\section{REFERENCES}

1. The Joint Commission. Improving America's Hospitals: The Joint Commission's Annual Report on Quality and Safety. Oak Brook, IL: Joint Commission Resources, 2007.

2. Arora VM, et al. Hospitalist handoffs: a systematic review and task force recommendations. J Hosp Med. 2009;4:433440 .

3. Riesenberg LA, Leitzsch J, Cunningham JM. Nursing handoffs: a systematic review of the literature. Am J Nurs. 2010;110:24-34

4. Kripalani S, et al. Deficits in communication and information transfer between hospital-based and primary care physicians: implications for patient safety and continuity of care. JAMA. 2007;297:831-841.

5. Hesselink $G$, et al. Improving patient handovers from hospital to primary care: a systematic review. Ann Intern Med. 2012;157:417-428.

6. Hilligoss B, et al. Collaborating-Or "selling" patients? A conceptual framework for emergency department-toinpatient handoff negotiations. Jt Comm J Qual Patient Saf. 2015;41:134-143.

7. Patterson ES, Wears RL. Patient handoffs: standardized and reliable measurement tools remain elusive. Jt Comm J Qual Patient Saf. 2010;36:52-61.

8. Hilligoss B, Cohen MD. The unappreciated challenges of between-unit handoffs: negotiating and coordinating across boundaries. Ann Emerg Med. 2013;61:155-160.

9. Pantilat $\mathrm{SZ}$, et al. Primary care physician attitudes regarding communication with hospitalists. Am J Med. 2001;111:15s20 s.

10. Solet DJ, et al. Lost in translation: challenges and opportunities in physician-to-physician communication during patient handoffs. Acad Med. 2005;80:1094-1099.

11. Mozaffarian D, et al. Heart disease and stroke statistics-2015 update: a report from the American Heart Association. Circulation. 2015;131:e29-e322.

12. Ntaios $G$, Bath $P$, Michel P. Blood pressure treatment in acute ischemic stroke: a review of studies and recommendations. Curr Opin Neurol. 2010;23:46-52.

13. Adams RJ, et al. Coronary risk evaluation in patients with transient ischemic attack and ischemic stroke: a scientific statement for healthcare professionals from the Stroke Council and the Council on Clinical Cardiology of the American Heart Association/American Stroke Association. Circulation. 2003;108:1278-1290.

14. Rudd AG, et al. Secondary prevention for stroke in the United Kingdom: results from the National Sentinel Audit of Stroke. Age Ageing. 2004;33:280-286. 
15. $\mathrm{Xu} \mathrm{G}$, et al. Recurrence after ischemic stroke in Chinese patients: impact of uncontrolled modifiable risk factors. Cerebrovasc Dis. 2007;23:117-120.

16. Lager $\mathrm{KE}$, et al. Interventions for improving modifiable risk factor control in the secondary prevention of stroke. Cochrane Database Syst Rev. 2014;(5):CD009103.

17. Kaplan RC, et al. Vascular events, mortality, and preventive therapy following ischemic stroke in the elderly. Neurology. 2005;65:835-842.

18. Steele DJ, Jackson TC, Gutmann MC. Have you been taking your pills? The adherence-monitoring sequence in the medical interview. J Fam Pract. 1990;30:294-299.

19. Heisler $M$, et al. When more is not better: treatment intensification among hypertensive patients with poor medication adherence. Circulation. 2008;117:2884-2892.

20. Frankel RM, Beckman HB. Conversation and compliance with medical recommendations: an application of micro-analysis in medicine. Rethink Commun. 1989;2:60-74.

21. Brown $\mathrm{SH}$, et al. VistA-U.S. Department of Veterans Affairs national-scale HIS. Int J Med Inform. 2003;69:135-156.

22. Weir CR, et al. Direct text entry in electronic progress notes. An evaluation of input errors. Methods Inf Med. 2003;42:6167.

23. Patterson E. Handoffs: lessons from other industries. OR Manager. 2005;21:11-12.

24. Taylor A, et al. Appreciating the power of narratives in healthcare: a tool for understanding organizational complexity and values. In: Candlin CN, Sarangi S, eds. Handbook of Communication in Organisations and Professions. Berlin: DeGruyter Mouton, 2011:457-480.

25. Willis GB. Cognitive Interviewing: A Tool for Improving Questionnaire Design. Thousand Oaks, CA: Sage, 2005.

26. Muhr T. Atlas.ti, version 6.2. Berlin: Scientific Software Development, 2010.

27. Borkan J. Immersion/crystallization. In: Crabtree BF, Miller WL, eds. Doing Qualitative Research. 2nd ed. Thousand Oaks, CA: Sage, 1999:179-194.

28. Miles MB, Huberman AM, Saldaña J. Qualitative Data Analysis: A Methods Sourcebook. 3rd ed. Thousand Oaks, CA: Sage, 2014.

29. Weis JM, Levy PC. Copy, paste, and cloned notes in electronic health records: prevalence, benefits, risks, and best practice recommendations. Chest. 2014;145:632638.

30. Rosland AM, et al. The patient-centered medical home in the Veterans Health Administration. Am J Manag Care. 2013;19:e263-e272.

31. Rodriguez HP, et al. Teamlet structure and early experiences of medical home implementation for veterans. J Gen Intern Med. 2014;29(suppl 2):S623-S631.

32. Davis MM, et al. Did I do as best as the system would let me?" Healthcare professional views on hospital to home care transitions. J Gen Intern Med. 2012;27:1649-1656.

33. Bell CM, et al. Association of communication between hospital-based physicians and primary care providers with patient outcomes. J Gen Intern Med. 2009;24:381-386.
34. van Walraven $C$, Rokosh $E$. What is necessary for high-quality discharge summaries? Am J Med Qual. 1999;14:160-169.

35. Nahab F, et al. Avoidable 30-day readmissions among patients with stroke and other cerebrovascular disease. Neurohospitalist. 2012;2:7-11.

36. Johnson JK, Arora VM, Barach PR. What can artefact analysis tell us about patient transitions between the hospital and primary care? Lessons from the HANDOVER project. Eur J Gen Pract. 2013;19:185-193.

37. Toccafondi $G$, et al. The collaborative communication model for patient handover at the interface between high-acuity and low-acuity care. BMJ Qual Saf. 2012;21(suppl 1):i58-i66.

38. Greysen SR, et al. "Out of sight, out of mind": housestaff perceptions of quality-limiting factors in discharge care at teaching hospitals. J Hosp Med. 2012;7:376-381.

39. Campbell EM, et al. Types of unintended consequences related to computerized provider order entry. J Am Med Inform Assoc. 2006;13:547-556.

40. Saleem JJ, et al. The next-generation electronic health record: perspectives of key leaders from the US Department of Veterans Affairs. J Am Med Inform Assoc. 2013;20:e175e177.

41. Toll E. The cost of technology. JAMA. 2012;307:2497-2498.

42. Embi PJ, et al. Impacts of computerized physician documentation in a teaching hospital: perceptions of faculty and resident physicians. J Am Med Inform Assoc. 2004;11:300-309.

43. Makary MA, Daniel M. Medical error-The third leading cause of death in the US. BMJ. 2016;353:i2139.

44. Legault K, et al. Quality of discharge summaries prepared by first year internal medicine residents. BMC Med Educ. 2012;12:77.

45. Bergman AA, et al. "Mr Smith's been our problem child today ...": anticipatory management communication (AMC) in VA end-of-shift medicine and nursing handoffs. BMJ Qual Saf. 2016;25:84-91.

46. Nowak MA. Five rules for the evolution of cooperation. Science. 2006;314:1560-1563.

47. Philibert I, Barach P. The European HANDOVER Project: a multi-nation program to improve transitions at the primary care-inpatient interface. BMJ Qual Saf. 2012;21(suppl 1):i1-i6.

48. Riesenberg LA, et al. Residents' and attending physicians' handoffs: a systematic review of the literature. Acad Med. 2009;84:1775-1787.

49. Myers JS, et al. Are discharge summaries teachable? The effects of a discharge summary curriculum on the quality of discharge summaries in an internal medicine residency program. Acad Med. 2006;81(10 suppl):S5-S8.

50. Hilligoss B, Cohen MD. Hospital handoffs as multifunctional situated routines: implications for researchers and administrators. Adv Health Care Manag. 2011;11:91-132.

51. Horwitz LI, et al. Validation of a handoff assessment tool: the Handoff CEX. J Clin Nurs. 2013;22:1477-1486.

52. Hilligoss B, Moffatt-Bruce SD. The limits of checklists: handoff and narrative thinking. BMJ Qual Saf. 2014;23:528-633. 